\title{
Konzeption von Kommunikation
}


Klaus Merten

\section{Konzeption von \\ Kommunikation}

Theorie und Praxis des strategischen Kommunikationsmanagements

Springer VS 
Klaus Merten

Münster, Deutschland

ISBN 978-3-658-01466-7

ISBN 978-3-658-01467-4 (eBook)

DOI $10.1007 / 978-3-658-01467-4$

Die Deutsche Nationalbibliothek verzeichnet diese Publikation in der Deutschen Nationalbibliografie; detaillierte bibliografische Daten sind im Internet über http://dnb.d-nb.de abrufbar.

\section{Springer VS}

(C) Springer Fachmedien Wiesbaden 2013

Das Werk einschließlich aller seiner Teile ist urheberrechtlich geschützt. Jede Verwertung, die nicht ausdrücklich vom Urheberrechtsgesetz zugelassen ist, bedarf der vorherigen $\mathrm{Zu}$ stimmung des Verlags. Das gilt insbesondere für Vervielfältigungen, Bearbeitungen, Übersetzungen, Mikroverfilmungen und die Einspeicherung und Verarbeitung in elektronischen Systemen.

Die Wiedergabe von Gebrauchsnamen, Handelsnamen, Warenbezeichnungen usw. in diesem Werk berechtigt auch ohne besondere Kennzeichnung nicht zu der Annahme, dass solche Namen im Sinne der Warenzeichen- und Markenschutz-Gesetzgebung als frei zu betrachten wären und daher von jedermann benutzt werden dürften.

Gedruckt auf säurefreiem und chlorfrei gebleichtem Papier

Springer VS ist eine Marke von Springer DE. Springer DE ist Teil der Fachverlagsgruppe Springer Science+Business Media.

www.springer-vs.de 


\section{Inhalt}

1 Strategische Konzeption: Gezielt Wirkungen erreichen ～. . . . . . 11

1.1 Beispiel: Wo ist der Hamburger Hauptbahnhof? . . . . . . . . . . . . 13

1.2 PR-Konzeption in 12 Schritten . . . . . . . . . . . . . . 16

1.3 Das Konzeptionswirrwarr . . . . . . . . . . . . . . . . . . 86

2 Zur Theorie ........................ 91

2.1 Strategie und Kommunikation . . . . . . . . . . . . . . . . . . 91

2.1.1 Strategie und Strategem . . . . . . . . . . . . . . . . . . . . 92

2.1.2 Planung und Strategie . . . . . . . . . . . . . . . . . . . . . 95

2.1.3 Strategische PR-Konzeption . . . . . . . . . . . . . 96

2.2 Relevante Ansätze der Wirkungsforschung . . . . . . . . . . . . . . 100

2.2.1 Das Stimulus-Response-Modell . . . . . . . . . . . . . . . . . 102

2.2.2 Agenda-Setting Approach . . . . . . . . . . . . . . . . . 110

2.2.3 Theorie des Meinungsführers . . . . . . . . . . . . . . 115

2.2.4 Reflexive Mechanismen . . . . . . . . . . . . . 116

2.2.5 Der Third-Person-Effect . . . . . . . . . . . . . . . . 119

3 Methoden ....................... 121

3.1 Entdeckungs-, Begründungs- und Verwertungszusammenhang .. . 121

3.2 Beobachtung ..................... 133

3.2.1 Varianten der Beobachtung . . . . . . . . . . . . . 134

3.2.2 Fehlerquellen ................... . 137 
3.3 Befragung . . . . . . . . . . . . . . . . . . . . 139

3.3.1 Schriftliche Befragung _. . . . . . . . . . . . . . 140

3.3.2 Mündliche Befragung . . . . . . . . . . . . . . . . . 146

3.3 .3 Fehlerquellen . . . . . . . . . . . . . . . . . . . . 148

3.4 Inhaltsanalyse . . . . . . . . . . . . . . . . . . . . . . . . . . 154

3.4.1 Ziele und Mittel der Inhaltsanalyse ～. . . . . . . . . . . . . 155

3.4.2 Varianten der Inhaltsanalyse . . . . . . . . . . . 156

3.4.3 Ablaufplan einer Inhaltsanalyse . . . . . . . . . . . . . . 159

4 Die Praxis der Konzeption ～. . . . . . . . . . . . . . . . . . . . . 167

4.1 Das Briefing - Basis jeder Konzeption ～. . . . . . . . . . . . . . . 167

4.2 Kein Lösungsprinzip für ein Lösungsprinzip ． . . . . . . . . . . . . 168

4.2 .1 Zielfindung . . . . . . . . . . . . . . 170

4.2.2 Ansatz, Lösungsprinzip, Methode . . . . . . . . . . . . . . . 173

4.2 .3 Kommunikative Ziele . . . . . . . . . . . . . . . . . . . . 176

4.2.4 Kommunikative Funktionen und Maßnahmen _ . . . . . . . 178

4.2 .5 Zwischenfazit . . . . . . . . . . . . . . . . . . . . . . 182

4.3 Wo es schief gehen kann: Stolpersteine und Risiken

bei der Entwicklung einer Konzeption ～. . . . . . . . . . . . 183

4.4 Kreative Maßnahmen . . . . . . . . . . . . . . . . . . . . . . . 211

4.5 Konzeptpräsentation . . . . . . . . . . . . . . . . . . 213

5 Das Controlling f . . . . . . . . . . . . . . . . . . . . . 217

5.1 Das formale Ablaufcontrolling . . . . . . . . . . . . . . . 217

5.2 Effect Controlling . . . . . . . . . . . . . . . . . . . . . . . . . 219

5.2.1 Nonmediale Resonanzanalyse . . . . . . . . . . . . . . . 219

5.2 .2 Medienresonanzanalyse . . . . . . . . . . . . . . 220

5.2.3 Typen von Medienresonanzanalysen _ . . . . . . . . . 222

5.2.4 Extramedia-Aanalyse . . . . . . . . . . . . . . 226

5.2.5 Funktionsweise von Medienresonanzanalysen ～. . . . . . . . 227

5.2.6 Ergebnisse von Medienresonanzanalysen ～. . . . . . . . . . . 230

5.2 .7 Medienresonanz in Krisen ～. . . . . . . . . . . . . . . . . 235

5.3 Communications Control (Wertschöpfung) . . . . . . . . . . . 243 
6 Gute und schlechte Beispiele _ . . . . . . . . . . . . . . 245

6.1 Weitere Beispiele . . . . . . . . . . . . . . . . . . . . . . . . 246

6.2 Grosse Checkliste für den Ablauf einer PR-Konzeption . . . . . . . . 287

6.3 Regeln und Ratschläge . . . . . . . . . . . . . . . . . 300

6.3 .1 Regeln . . . . . . . . . . . . . . . . . 301

6.3 .2 Zwölf goldene Ratschläge $\ldots \ldots \ldots 303$

7 Glossar . . . . . . . . . . . . . . . . . . 305

Literaturverzeichnis . . . . . . . . . . . . . . . . . . 423

Stichwortverzeichnis . . . . . . . . . . . . . . 433 


\section{Einleitung}

Seit der Jahrtausendwende dominieren zwei Trends das gesellschaftliche (wirtschaftliche, politische) Handeln: Die Ausdehnung des strategischen Managements auf immer neue Handlungsfelder (etwa: Zeitmanagement, Krankenhausmanagement, Personalmanagement) und die Ausdehnung kommunikativen Handelns auf immer mehr Bereiche des Managements. Der erste Trend verdankt sich dem globalen Streben nach Optimierung des Einsatzes von Zeit- und Ressourcen aller Art, der zweite der Tatsache, dass Kommunikation eine maximal vorteilhafte Ressource darstellt, die sich überall einsetzen lässt, weil Kommunikation als genereller Stellvertreter für alles Handeln fungiert. Oder anders: Sage mir, wie erfolgreich Deine Kommunikation ist und ich sage Dir, wie erfolgreich Dein Management ist.

Management von Kommunikation beruht auf der Anfertigung einer strategischen Konzeption für Kommunikation und diese gilt mittlerweile als der Königsweg aller PR und allen Managements und zugleich als beinharter Test auf Kommunikations-Kompetenz: Hier müssen ein gerütteltes Maß an theoretischem Wissen, praktische Kenntnisse und vor allem große Erfahrung abgerufen und unter strategischer Perspektive sinnvoll genutzt werden.

Die vorliegende Einführung versucht, theoretisches Wissen und praktische Kenntnisse zur strategischen Kommunikations-Konzeption an zahlreichen Beispielen plausibel zu vermitteln. Der Leser soll nach der Durcharbeitung in der Lage sein, eine strategisch aufgebaute Kommunikations-Konzeption regelgerecht zu entwickeln und dabei alle Möglichkeiten der Optimierung zu nutzen.

Zunächst wird ein schlüssiger, theoretisch abgeleiteter Ablaufplan vorgestellt, anhand dessen beispielhaft von Mal zu Mal aufwendigere und schwierigere Konzeptionen mit ihrer jeweiligen Problemstellung entwickelt werden, die den strategischen Einsatz von Kommunikation sicherstellen. Damit legen wir die Basis für das Verständnis und die Entwicklung einer Konzeption und die Sicherheit zu deren professioneller Ausgestaltung. Die Diskussion guter und weniger guter Bei- 
spiele hilft, das Verständnis für die erfolgreiche Anfertigung einer Konzeption zu schärfen. Die jeweils getroffenen konzeptionellen Entscheidungen auf der Ebene der Situationsanalyse, der Strategie, der Taktik, der Umsetzung und des Controlling werden begründet, mögliche Alternativen diskutiert, Probleme und Gefahren aufgezeigt.

Das dafür notwendige, relevante Wissen über Wirkungen der Medien und der zu deren Messung eingesetzten Methoden wird in eigenen Kapiteln nachvollziehbar vermittelt. Ein umfassendes Glossar, ein Verzeichnis der Literatur und der wichtigsten Stichworte schließen sich an. 